\title{
Effect of mechanical relaxation time in elastic materials with voids
}

\author{
R. Lianngenga*, L. Thangmawia \\ Department of Mathematics, Pachhunga University College, Aizawl 796001, Mizoram, India
}

\begin{abstract}
The effect of mechanical relaxation time in the elastic wave propagation in elastic materials with voids is investigated. The phase speed and the attenuation coefficients are obtained and observed the effect of mechanical relaxation time. The phenomenon of reflection of elastic waves due to the incident waves from a plane boundary of elastic materials with voids is studied. The amplitude and energy ratios of the reflected waves are obtained. Numerically these ratios, phase speeds and the corresponding attenuation coefficients are computed for a particular model and the effect of mechanical relaxation time is discussed.
\end{abstract}

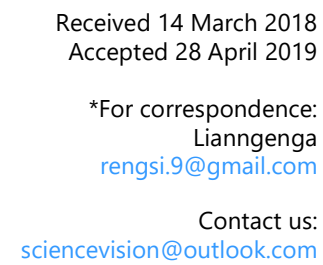

Keywords: Attenuation coefficient, phase speed, amplitude ratio, energy ratio, voids.

\section{Introduction}

Cowin and Nunziato presented a theory of linear elastic materials with voids. ${ }^{1}$ In this theory, the change in voids volume fraction and strain are taken as an independent kinematic variable. In fact, this theory is one of the generalisations of classical elasticity. The intended application of the theory of elastic materials with voids are found in geological materials like rocks, soils and many manufactured materials available in our environment such as wood, clothes, paper etc. Puri and Cowin analyzed the behaviour of plane harmonic waves in linear elastic materials with voids. ${ }^{2}$ They presented the existence of two longitudinal waves in this theory, both these waves are attenuate in their direction of propagation. The linear theory of homogeneous and isotropic materials with voids and initial boundary value problem in terms of stress and volume fraction fields is formulated by Chandrasekhariah. ${ }^{3}$

lovane and Nasedkin investigated the eigenvalue problems in elastic bodies with voids in contact with massive rigid plane punches using Cowin-Nunziato theory and they proved the discreteness of the spectrum and completeness of the eigenfunctions. ${ }^{4}$ Antonio Scalia studied and derived the jump conditions and growth equation which govern the propagation of shock waves in visco-elastic materials with voids. ${ }^{5}$ Adina Chirila introduced the Caputo fractional derivatives and applied for the solutions of problems on micropolar thermoelasticity and he incorporated the mechanical relaxation time in the context of constitutive and field equations. ${ }^{6}$ Scalia and Sumbatyan studied a static contact problem about a rigid punch on the free surface of linear porous elastic half-plane. ${ }^{7}$ They reduce the contact problem to a singular integral equation and described a direct numerical co-location technique to solve the problems.

The reflection of incident plane waves from a free boundary of porous elastic half-space was studied by Ciarletta and Sumbatyan. ${ }^{8}$ They have shown that only the transverse wave can propagate on the solid half-space without attenuation. The reflection and transmission due to the incident plane longitudinal waves at a plane interface between two elastic media with voids is investigated by Tomar and Singh. ${ }^{9}$ They found that the presences of voids are significant on 
reflection and transmission coefficients. Later, Singh and $\operatorname{Tomar}^{10}$ investigated the reflection and refraction of transverse wave incident obliquely at a plane interface between two porous elastic half spaces in welded contact. Chanrasekhariah investigated Rayleigh waves and Rayleigh-Lamb wave propagating at a plane material boundary of an elastic half-space containing a distribution of voids. ${ }^{11,12}$ He found that the waves are generally dispersive and the voids have no influence on the motion of Rayleigh wave for incompressible body.

Abo-Dahab and Singh studied the influence of rotation, magnetic field, voids and initial stress on the reflection of $P$ - waves in thermoelasticity without energy dissipation. ${ }^{13}$ Dey et al. analysed the possibility of surface torsion wave in an elastic halfspace with void pores, they found two types of surface waves, one is depending only on the parameters associated with change in void volume pores whereas the other depend on elastic constants of the half-spaces as well as on change of void volume parameters. ${ }^{14}$ Chirita and Ghiba presented inhomogeneous plane wave solution within the context of linear theory of poroelastic materials. ${ }^{15}$ The remarkable waves and vibrations are found in Achenbach, ${ }^{16} \quad$ Straughan, ${ }^{17}$ Lianngenga, ${ }^{18,19}$ Lianngenga et al., ${ }^{20,21}$ and Ciarletta and lesan. ${ }^{22}$

In this paper we incorporate the idea of Chirila ${ }^{6}$ into the theory of Cowin-Nunziato. ${ }^{1}$ Then, the effects of mechanical relaxation time in the propagations of elastic waves are investigated. The phase speed and attenuation coefficients of plane waves are obtained. And the amplitude and energy ratios of the reflected waves are found with the appropriate boundary conditions. Numerically and analytically the phase speeds, attenuation coefficients, amplitude and energy ratios are computed for a particular materials model.

\section{Basic Equations and Solutions}

Following Adina Chirila ${ }^{6}$ and Cowin and Nunziato, ${ }^{1}$ the constitutive equations including mechanical relaxation time for homogeneous isotropic elastic materials with voids in the absence of body forces can be written as

$$
\begin{aligned}
& \mathrm{t}_{\mathrm{ij}}=\lambda \delta_{\mathrm{ij}} \mathrm{DE}_{\mathrm{kl}}+2 \mu \mathrm{DE}_{\mathrm{kl}}+\beta \delta_{\mathrm{ij}} \phi \\
& \mathrm{g}=-\mathrm{b} \dot{\phi}-\xi \phi-\beta D E_{\mathrm{kk}} \\
& \mathrm{h}_{\mathrm{i}}=\alpha \phi \ldots \ldots \ldots \ldots
\end{aligned}
$$

where $\left(t_{i j}, h_{i}, g\right)$ are components of stress tensor, equilibrated stress vector, intrinsic equilibrated body force respectively; $(\lambda, \mu)$ are lame's constants; $(\alpha, \beta, \xi, b)$ are voids parameters; $\mathrm{E}_{\mathrm{kl}}=$ $\frac{1}{2}\left(\mathrm{u}_{\mathrm{i}, \mathrm{j}}+\mathrm{u}_{\mathrm{j}, \mathrm{i}}\right) ; \mathrm{u}_{\mathrm{i}}\left(\mathrm{x}_{\mathrm{i}}, \mathrm{t}\right)-$ components of displacement and $\phi\left(\mathrm{x}_{\mathrm{i}}, \mathrm{t}\right)-$ voids volume fraction; $\left(\mathrm{x}_{\mathrm{i}}, \mathrm{t}\right)$ are components of spatial position vector in Cartesian coordinates system $\mathrm{x}_{1}, \mathrm{x}_{2}, \mathrm{x}_{3}$ and time variable respectively; $\tau-$ mechanical relaxation time, $\mathrm{D}=1+$ where $\left(t_{i j}, h_{i}, g\right)$ are components of stress tensor, equilibrated stress vector, intrinsic equilibrated body force respectively; $(\lambda, \mu)$ are lame's constants; $(\alpha, \beta, \xi, \mathrm{b})$ are voids parameters; $\mathrm{E}_{\mathrm{kl}}=$ $\frac{1}{2}\left(\mathrm{u}_{\mathrm{i}, \mathrm{j}}+\mathrm{u}_{\mathrm{j}, \mathrm{i}}\right) ; \mathrm{u}_{\mathrm{i}}\left(\mathrm{x}_{\mathrm{i}}, \mathrm{t}\right)$ - components of displacement and $\phi\left(\mathrm{x}_{\mathrm{i}}, \mathrm{t}\right)-$ voids volume fraction; $\left(\mathrm{x}_{\mathrm{i}}, \mathrm{t}\right)$ are components of spatial position vector in Cartesian coordinates system $\mathrm{x}_{1}, \mathrm{x}_{2}, \mathrm{x}_{3}$ and time variable respectively; $\tau-$ mechanical relaxation time, $\mathrm{D}=1+$ $\tau^{r} D_{t}^{r}$ and $D_{t}^{r}$ is a fractional order derivative with respect to time; the superpose dot implies time derivatives and ()$_{, \mathrm{i}}=\partial() / \partial \mathrm{x}_{\mathrm{i}}$.

For simplicity, we shall take $r=1$ throughout the article, and then the fractional order derivative $\mathrm{D}_{\mathrm{t}}^{\mathrm{r}}$ will transform into $\frac{\partial}{\partial \mathrm{t}}$ and $\mathrm{D}=1+\tau \frac{\partial}{\partial \mathrm{t}}$.

The field equation for homogeneous isotropic elastic materials with the mechanical relaxation time in the absence of body forces are given as

$\mu \mathrm{D} \nabla^{2} \mathbf{u}+(\lambda+\mu) \mathrm{D} \nabla \nabla \cdot \mathbf{u}+\beta \nabla \phi=\rho \ddot{\mathbf{u}}$

$\alpha \nabla^{2} \phi-\xi \phi-b \dot{\phi}-\beta D \nabla \cdot \mathbf{u}=\rho \mathrm{k} \ddot{\phi}$

where $\nabla=\left(\partial / \partial \mathrm{x}_{1}, \partial / \partial \mathrm{x}_{2}, \partial / \partial \mathrm{x}_{3}\right)$.

The constitutive and the field equation may satisfy the following conditions (see Cowin-Nunziato ${ }^{1}$ )

$$
\mu, \alpha, \xi \geq 0 ; 3 \lambda+2 \mu \geq 0 ;(3 \lambda+2 \mu) \xi \geq 12 \beta^{2}
$$

Using Helmholtz decomposition theorem, we can split the displacement vector (u) into the scalar and vector potential as

$$
\mathbf{u}=\nabla \mathrm{p}+\nabla \times \mathbf{q}, \nabla \cdot \mathbf{q}=0
$$

where $p$ is scalar potential of $\mathbf{u}$ and $\mathbf{q}$ is vector potential of $\mathbf{u}$.

Using Helmholtz decomposition theorem (6) into the field equation (4)-(5), we obtain

$$
\begin{aligned}
& \mu D \nabla^{2} p+(\lambda+\mu) D \nabla^{2} p+\beta \phi=\rho \ddot{p} \\
& \alpha \nabla^{2} \phi-\xi \phi-b \dot{\phi}-\beta D \nabla^{2} p=\rho k \ddot{\phi} \\
& \mu D \nabla^{2} q=\rho \ddot{q} w i t h q=(0, q, 0)
\end{aligned}
$$

In order to discuss the wave propagation in linear homogeneous elastic materials with voids, we consider the plane harmonic travelling wave with

$\{\mathrm{p}, \phi, \mathrm{q}\}=\left\{\mathrm{p}_{0}, \phi_{0}, \mathrm{q}_{0}\right\} \mathrm{e}^{-\mathrm{i} \omega \mathrm{t}}$ where $\mathrm{i}=\sqrt{-1}, \omega(=\mathrm{kc})$ is angular frequency, $\mathrm{k}-$ is wavenumber and $\mathrm{c}-$ phase speed.

Applying equation (10) into the equations (7)-(9) we get

$$
\begin{aligned}
& \left(c_{1}^{2} D \nabla^{2}+\omega^{2}\right) p_{0}+c_{2}^{2} \phi_{0}=0 \\
& c_{3}^{2} D \nabla^{2} p_{0}-\left(c_{4}^{2} \nabla^{2}+c_{5}^{2}\right) \phi_{0}=0 \\
& \left(\mu D \nabla^{2}+\rho \omega^{2}\right) q_{0}=0
\end{aligned}
$$
where $c_{1}^{2}=\frac{\lambda+\mu}{\rho}, c_{2}^{2}=\frac{\beta}{\rho}, c_{3}^{2}=\frac{\beta}{\rho k^{\prime}}, c_{4}^{2}=\frac{\alpha}{\rho \mathrm{k}}, c_{5}^{2}=\frac{\mathrm{i} \omega \mathrm{b}-\xi}{\rho \mathrm{k}}+$ $\omega^{2}$. 
Equations (11) and (12) are couple inp $\mathrm{p}_{0}$ and $\phi_{0}$ they represent coupled longitudinal waves while equation (13) represent shear wave. On solving equations (11) and (12) for the non-trivial solutions, we obtain the frequency equation for coupled longitudinal wave as

$$
A \nabla^{4}+\omega^{2} B \nabla^{2}+\omega^{4} C=0
$$

where $\mathrm{A}=\mathrm{c}_{1}^{2} \mathrm{c}_{4}^{2} \mathrm{D}, \mathrm{B}=\mathrm{c}_{1}^{2} \mathrm{c}_{5}^{2} \mathrm{D} / \omega^{2}+\mathrm{c}_{2}^{2} \mathrm{c}_{5}^{2} / \omega^{2}+\mathrm{c}_{4}^{2}, \mathrm{C}=$ $c_{5}^{2} / \omega^{2}$.

Following Helmholtz equation

$\left(\nabla^{2}+\mathrm{k}_{\mathrm{i}}\right)\left\{\mathrm{p}_{0}, \phi_{0}, \mathrm{q}_{0}\right\}=0, \mathrm{k}_{\mathrm{i}}=\frac{\omega}{c_{\mathrm{i}}} \mathrm{T}$

Thus, using Equation (15) into (14) we obtain the solutions of the two coupled longitudinal waves in terms of wave number as

$$
\mathrm{k}_{1,2}^{2}=\frac{\omega^{2}\left(\mathrm{~B} \pm \sqrt{\mathrm{B}^{2}-4 \mathrm{AC}}\right)}{2 \mathrm{~A}}
$$

Similarly, equation (13) gives the solution for shear wave as

$$
\mathrm{k}_{3}^{2}=\frac{\rho \omega^{2}}{\mu \mathrm{D}}
$$

Since the quadratic equation (14) and linear equation (13) has complex coefficients, their roots will be complex value. Thus, the coupled longitudinal and shear wave are attenuating in nature. The phase speeds $\left(\mathrm{c}_{\mathrm{i}}\right)$ and attenuation coefficients $\left(\mathrm{A}_{\mathrm{i}}\right)$ of the elastic waves in homogeneous isotropic materials with voids are obtained as

$$
c_{i}=\frac{\omega}{\operatorname{Re}\left(k_{i}\right)} \text {, and }\left|A_{i}\right|=-\operatorname{Im}\left(k_{i}\right)
$$

where $\operatorname{Re}()$ and $\operatorname{Im}()$ are real and imaginary part of () .

\section{Reflections from Boundary Surface}

In this section, our aim is to analyse the reflection phenomenon of an incident plane longitudinal wave from the plane boundary surface of homogeneous isotropic half-space, $\mathrm{H}=\{(\mathrm{x}, \mathrm{z}) ;-\infty<x<\infty, z \geq 0\}$, of elastic materials with voids. We shall assume that the train of elastic wave is originated from infinity and striking obliquely the plane boundary surface. We shall assume that the surface is free from all kinds of stresses and we shall assume the plane strain parallel to the xz-plane only.

Now we shall assume the following

define as

$$
\mathbf{u}=\left(\mathrm{u}_{1}, 0, \mathrm{u}_{3}\right) ; \phi=\phi(\mathrm{x}, \mathrm{z})
$$

$$
\mathrm{u}_{1}=\frac{\partial \mathrm{p}}{\partial \mathrm{x}}-\frac{\partial \mathrm{q}}{\partial \mathrm{z}} ; \mathrm{u}_{3}=\frac{\partial \mathrm{p}}{\partial \mathrm{z}}+\frac{\partial \mathrm{q}}{\partial \mathrm{x}}
$$

The incident plane waves at the boundary surface of the considered half-space reflected the other three elastic waves, two coupled longitudinal and one shear wave.
The boundary half-space $(H)$ at $z=0$ is free from all kind of stresses/tractions. Mathematically the condition can be written as

$$
\mathrm{t}_{\mathrm{zz}}=0, \mathrm{t}_{\mathrm{zx}}=0, \mathrm{~h}_{3}=0
$$

which are expressed as

$$
\begin{aligned}
& \lambda \mathrm{D}\left(\frac{\partial^{2} \mathrm{p}}{\partial \mathrm{x}^{2}}+\frac{\partial^{2} \mathrm{p}}{\partial \mathrm{z}^{2}}\right)+2 \mu \mathrm{D}\left(\frac{\partial^{2} \mathrm{p}}{\partial \mathrm{z}^{2}}+\frac{\partial^{2} \mathrm{q}}{\partial \mathrm{x} \partial \mathrm{z}}\right)+\beta \phi \\
& =0,---(20) \\
& 2 \frac{\partial^{2} \mathrm{p}}{\partial \mathrm{x} \partial \mathrm{z}}+\frac{\partial^{2} \mathrm{q}}{\partial \mathrm{x}^{2}}-\frac{\partial^{2} \mathrm{q}}{\partial \mathrm{z}^{2}}=0 ; \frac{\partial \phi}{\partial \mathrm{z}}=0
\end{aligned}
$$

The potentials $\{p, \phi, q\}$ in the half-space $(H)$ are written as

$$
\begin{gathered}
\mathrm{p}=\mathrm{A}_{0} \exp \left(\mathrm{P}_{0}\right)+\mathrm{A}_{1} \exp \left(\mathrm{P}_{1}\right)+\mathrm{A}_{2} \exp \left(\mathrm{P}_{2}\right) \ldots \ldots \ldots . .(22) \\
\phi=\mathrm{A}_{0} \sigma_{1} \exp \left(\mathrm{P}_{0}\right)+\mathrm{A}_{1} \sigma_{1} \exp \left(\mathrm{P}_{1}\right)+\mathrm{A}_{2} \sigma_{2} \exp \left(\mathrm{P}_{2}\right)
\end{gathered}
$$

$$
\mathrm{q}=\mathrm{A}_{3} \exp \left(\mathrm{P}_{3}\right)
$$

where $\quad P_{0}=i k_{0}\left(x_{1} \sin \theta_{0}-x_{3} \cos \theta_{0}-c_{0} t\right), \quad P_{i}=$ $\mathrm{ik}_{\mathrm{i}}\left(\mathrm{x}_{1} \sin \theta_{\mathrm{i}}+\mathrm{x}_{3} \cos \theta_{\mathrm{i}}-\mathrm{c}_{\mathrm{i}} \mathrm{t}\right) ;\left(\mathrm{c}_{0}, \mathrm{k}_{0}\right)$ are the incident longitudinal phase speed and wavenumber respectively; $A_{0}$ is the incident amplitude constant of longitudinal waves and $A_{i}(i=1,2)$ is the amplitude constant of the reflected waves; $\sigma_{\mathrm{i}}=\left(\omega^{2}-\mathrm{c}_{1}^{2} \mathrm{Dk}_{\mathrm{i}}\right) / \mathrm{c}_{2}^{2}$ is the coupling constants; $\left(\theta_{0}, \theta_{i}\right)$ are the incident angle and reflected angles respectively.

The Snell's law used for this problem is

$\mathrm{k}_{0} \sin \theta_{0}=\mathrm{k}_{1} \sin \theta_{1}=\mathrm{k}_{2} \sin \theta_{2}=\mathrm{k}_{3} \sin \theta_{3} \ldots . .$. (25)

It may worth to note that the angle of incidence is equal to the angle of reflected for a particular wave.

Using equations (22)-(25) into the (20) and (21), we obtain the following system of equations as

$$
\left(\begin{array}{lll}
a_{11} & a_{12} & a_{13} \\
a_{21} & a_{22} & a_{23} \\
a_{31} & a_{32} & a_{33}
\end{array}\right)\left(\begin{array}{l}
\mathrm{Z}_{1} \\
Z_{2} \\
Z_{3}
\end{array}\right)=\left(\begin{array}{l}
b_{1} \\
b_{2} \\
b_{3}
\end{array}\right)
$$

where $a_{1 r}=\left(\lambda D+2 \mu D \cos ^{2} \theta_{r}-\beta \sigma_{r}\right) k_{r}^{2}, \quad(r=$ $1,2) \quad ; \quad a_{13}=2 \mu \mathrm{D} \cos \theta_{3} \sin \theta_{3} \mathrm{k}_{3}^{2} \quad ; \quad \mathrm{a}_{2 \mathrm{r}}=$ $-2 \mu \mathrm{D} \cos \theta_{\mathrm{r}} \sin \theta_{\mathrm{r}} \mathrm{k}_{\mathrm{r}}^{2},(\mathrm{r}=1,2) ; \mathrm{a}_{23}=\left(\cos ^{2} \theta_{3}-\right.$ $\left.\sin ^{2} \theta_{3}\right) k_{3}^{2} ; a_{3 r}=\sigma_{r} \cos \theta_{r} k_{r},(r=1,2) ; a_{33}=0 ; b_{1}=$ $-a_{11} ; b_{2}=a_{21} ; b_{3}=a_{31} ; z_{r}=A_{r} / A_{0},(r=1,2,3)$.

By solving equation (26), we shall obtain the amplitude ratios. If the incident wave is shear wave then $\left(c_{0}, A_{0}\right)$ represent for shear wave with $b_{1}=\mathrm{a}_{13}$, $b_{2}=-\mathrm{a}_{23}$ and $b_{2}=\mathrm{a}_{33}$.

\section{Energy Ratios}

Consider the energy partitions $\left(E^{*}\right)$, the rate of energy transmission at the surface $(z=0)$ per unit area per unit time is given by

$$
\mathrm{E}^{*}=\left\langle\mathrm{t}_{33}, \dot{\mathrm{u}}_{3}\right\rangle+\left\langle\mathrm{t}_{31}, \dot{\mathrm{u}}_{1}\right\rangle+\left\langle\mathrm{h}_{3}, \dot{\phi}\right\rangle
$$

The energy of incident longitudinal waves is given as

$$
E_{\text {inc }}=\left\{(\lambda+2 \mu) D-\frac{\sigma_{1}}{k_{1}^{2}}\right\} \omega k_{1}^{3} \cos \theta_{1} A_{1}^{2} \exp \left\{2 P_{0}\right\}
$$


The energy ratios $\left(E_{i}, i=1,2,3\right)$ of various reflected waves are

$$
\begin{aligned}
& E_{1}=Z_{1}^{2}, \\
& E_{2}=\frac{\left\{(\lambda+\mu) D-\frac{\beta \sigma_{2}}{k_{2}^{2}}\right\} k_{2}^{3} \cos \theta_{2}}{\left\{(\lambda+\mu) D-\frac{\beta \sigma_{1}}{k_{1}^{2}}\right\} k_{1}^{3} \cos \theta_{1}} Z_{2}^{2} \\
& E_{3}=\frac{\mu k_{3}^{3} \cos \theta_{3}}{\left\{(\lambda+\mu) D-\frac{\beta \sigma_{1}}{k_{1}^{2}}\right\} k_{1}^{3} \cos \theta_{1}} Z_{2}^{2}
\end{aligned}
$$

\section{Results and Discussion}

In order to understand the nature of dependence of phase speeds on angular frequency and the nature of dependence of reflection coefficients (amplitude and energy ratios) on angle of incidence, we have computed them numerically for a particular model using MATLAB software. The phase speeds and attenuation coefficients are computed from the Equation (18) while the amplitude and energy ratios are computed using equations (26) and (29)-(31) respectively. For this purpose, the relevant values of the following parameters are considered:

It is seen that there are two coupled longitudinal waves that are assigning the fast-longitudinal wave $\left(c_{1}\right)$ and slow longitudinal wave $\left(c_{2}\right)$. The dependence of phase speeds and attenuation coefficients on the angular frequency $(\omega)$ are shown in Figures 1-6. And the dependence of Amplitude and energy ratios on incident angle $\left(\theta_{0}\right)$ are shown in Figures 7-12. All the Figures show that the effect of mechanical relaxation time $(\tau)$, Curve I indicate the value of $\tau=0.013 \mathrm{~s}$ and Curve II indicate the value $\tau=0$.

In Figure 1-2, the phase speeds of fast and slow longitudinal waves $\left(c_{1}\right.$ and $c_{2}$ ) increase as increasing angular frequency, and the absence of mechanical relaxation time $(\tau)$ show higher value than the normal values. In Figure 3, the phase speed of shear wave $\left(c_{3}\right)$ starts from certain value and increase as increasing angular frequency while the absence of mechanical relaxation time shows constant speed for the shear wave.

The corresponding attenuation coefficients are shown in Figure 4-6. The attenuation coefficient of fast longitudinal wave $\left(\left|A_{1}\right|\right)$ is seen in Figure 4, it starts increasing from certain value upto the maximum and then decrease as increasing angular frequency. In Figure 5, the attenuation coefficient of slow longitudinal wave $\left(\left|A_{2}\right|\right)$ starts decrease from certain value as increasing angular frequency. The absence of mechanical relaxation time (i.e. $\tau=0$ ) has shown higher value than the normal value (i.e. $\tau=$
$0013 s)$. It is also seen that the value of $\left|A_{1}\right|$ is lower than $\left|A_{2}\right|$. It is seen in the Figure 6 that there is no attenuation for the shear wave in the absence of mechanical relaxation time while the normal shear wave attenuation coefficient $\left|A_{3}\right|$ increase as increasing angular frequency.

The amplitude ratios for incident fast-longitudinal wave are depicted in Figure 7-9 while the energy ratios are depicted in Figure 10-12. We have plotted the amplitude and energy ratios at the angular frequency of $5 s^{-1}$. In Figure 7, the amplitude ratio of reflected fast-longitudinal wave $\left(\left|Z_{1}\right|\right)$ increase as increasing angle of incidence $\left(\theta_{0}\right)$ while the amplitude ratio $\left|Z_{2}\right|$ of slow longitudinal wave decrease as increasing angle of incidence, seen in Figure 8 . The amplitude ratio $\left|Z_{3}\right|$ of shear wave has shown parabolic nature between the normal $\left(\theta_{0}=0^{\circ}\right)$ and grazing $\left(\theta_{0}=90^{\circ}\right)$ angle of incidence as shown in Figure 9. The amplitude ratio in the absence of mechanical relaxation time has lower value than the normal amplitude ratios.

The energy ratios for incident fast-longitudinal waves are found in Figure 10-12. The energy ratio of fast-longitudinal wave $\left(\left|E_{1}\right|\right)$, in Figure 10, increase from certain value upto the grazing angle of incidence. And the ratio $\left|E_{2}\right|$ of slow longitudinal wave decrease as increasing angle of incidence as shown in Figure 11. In Figure 12, the shear wave energy ratio $\left(\left|E_{3}\right|\right)$ also show parabolic nature between the normal and grazing angle of incidence. The normal mechanical relaxation time show higher values than the absence throughout the energy ratios. It is also noted that the curves II of the energy ratios, $\left|E_{2}\right|$ and $\left|E_{3}\right|$ of slow longitudinal and shear wave, are multiplied by 0.9 to show more clearly the effect of mechanical relaxation time.

\section{Conclusions}

The effect of thermal relaxation time in the elastic wave propagation in the homogeneous isotropic elastic materials with voids has been investigated. The formula for the phase speeds and the corresponding attenuation coefficients are obtained. The reflection phenomenon of incident fast-longitudinal wave at the boundary surface has also been studied and the amplitude and energy ratios are found. Numerically these observations are calculated for a particular material model to see the effect of mechanical relaxation time. The following are the conclusions: 
Table 1 | Value of parameters.

\begin{tabular}{ccc}
\hline Symbol & Value & Unit \\
\hline $\boldsymbol{\lambda}$ & $7.59 \times 10^{10}$ & $\mathrm{~N} / \mathrm{m}^{2}$ \\
$\boldsymbol{\mu}$ & $1.89 \times 10^{10}$ & $\mathrm{~N} / \mathrm{m}^{2}$ \\
$\boldsymbol{\rho}$ & $2.19 \times 10^{3}$ & $\mathrm{Kg} / \mathrm{m}^{3}$ \\
$\boldsymbol{\alpha}$ & $1 \times 10^{-10}$ & $\mathrm{~N}$ \\
$\boldsymbol{\beta}$ & $1.02 \times 10^{10}$ & $\mathrm{~N} / \mathrm{m}^{2}$ \\
$\boldsymbol{\xi}$ & $1.475 \times 10^{10}$ & $\mathrm{~N} / \mathrm{m}^{2}$ \\
$\boldsymbol{b}$ & $0.25 \times 10^{10}$ & $\mathrm{Ns} / \mathrm{m}^{2}$ \\
$\boldsymbol{\tau}$ & 0.013 & $\mathrm{~s}$ \\
$\boldsymbol{k}$ & 0.00753 & $\mathrm{~m}^{2}$
\end{tabular}

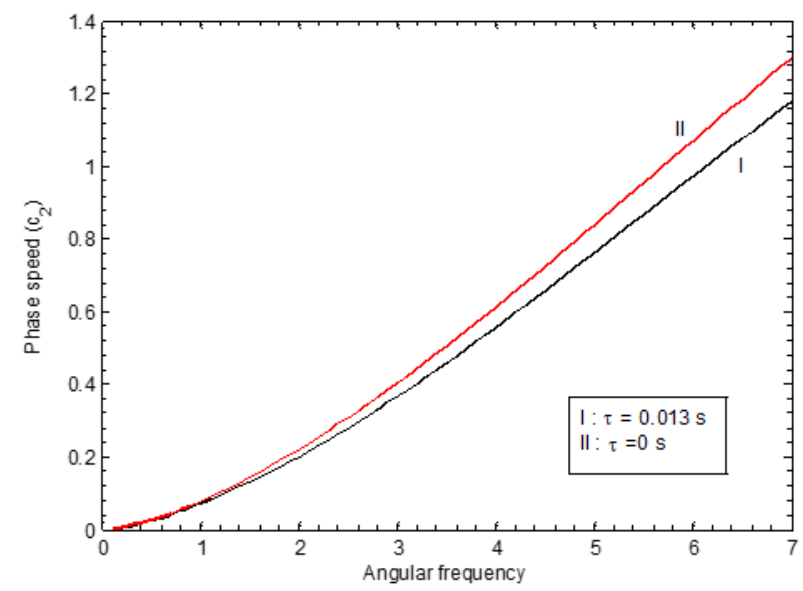

Figure 2 | Phase speed of slow longitudinal wave $\left(C_{2}\right)$.

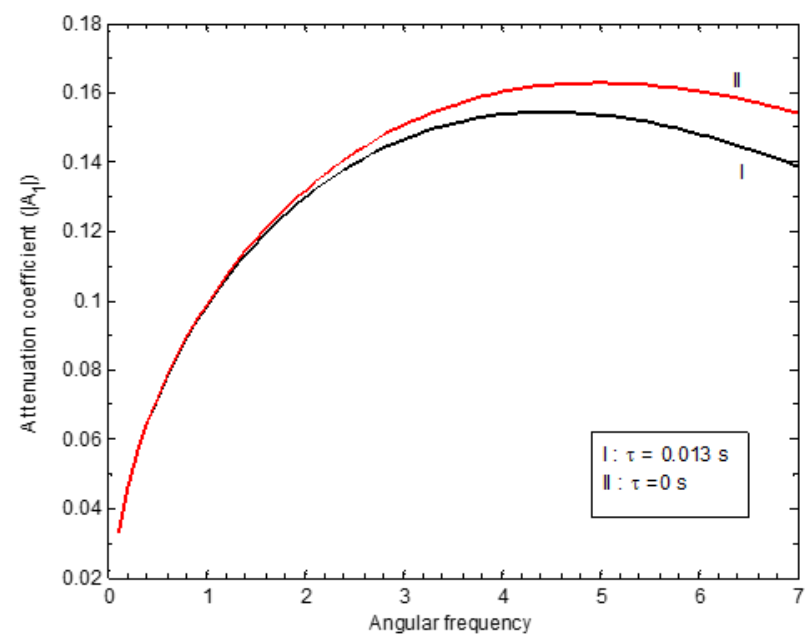

Figure 4 | Attenuation coefficient of fast longitudinal wave $\left(\left|A_{1}\right|\right)$.

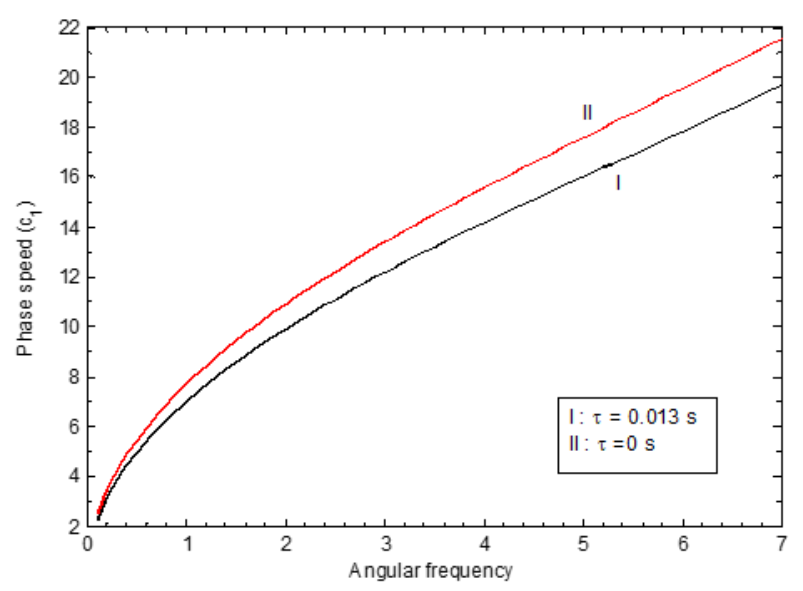

Figure 1 | Phase speed of fast longitudinal wave $\left(C_{1}\right)$.

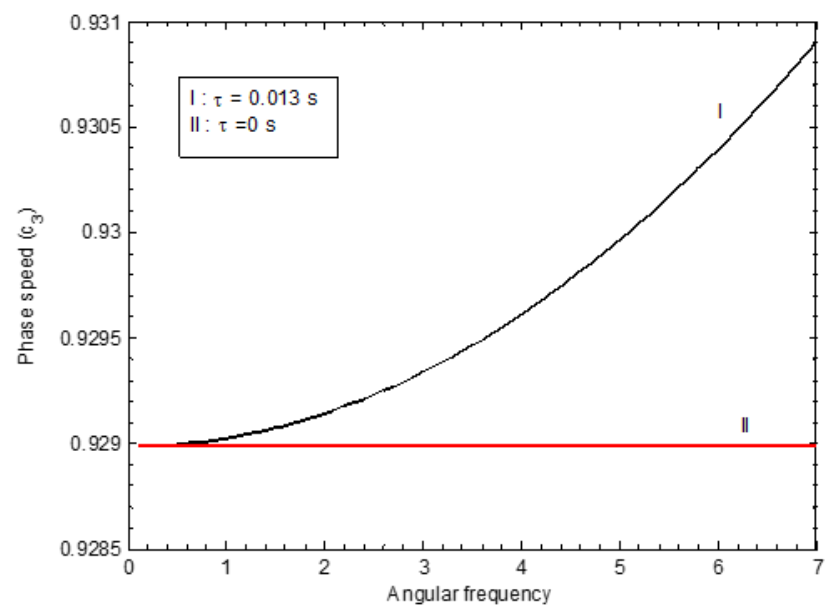

Figure 3 | Phase speed of shear wave $\left(C_{3}\right)$.

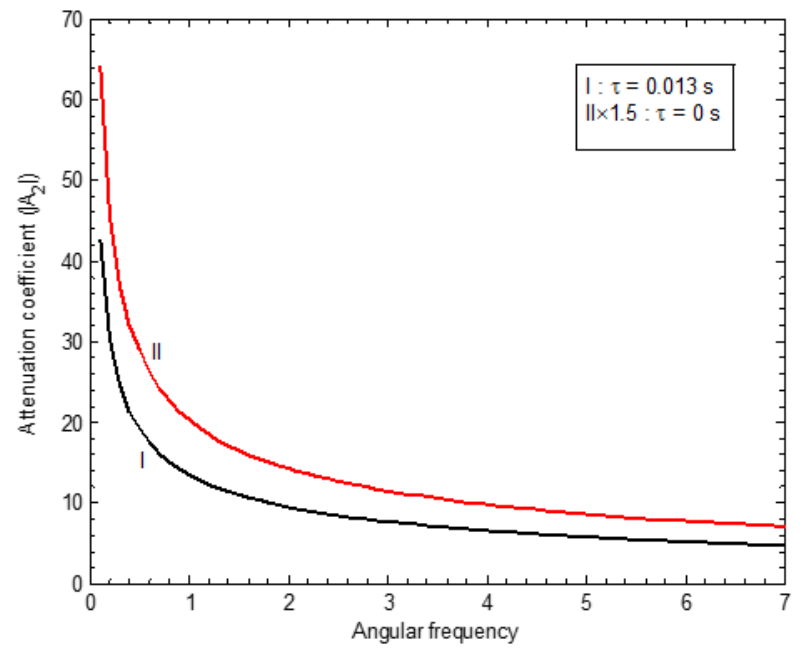

Figure 5 | Attenuation coefficient of slow longitudinal wave $\left(\left|A_{2}\right|\right)$. 


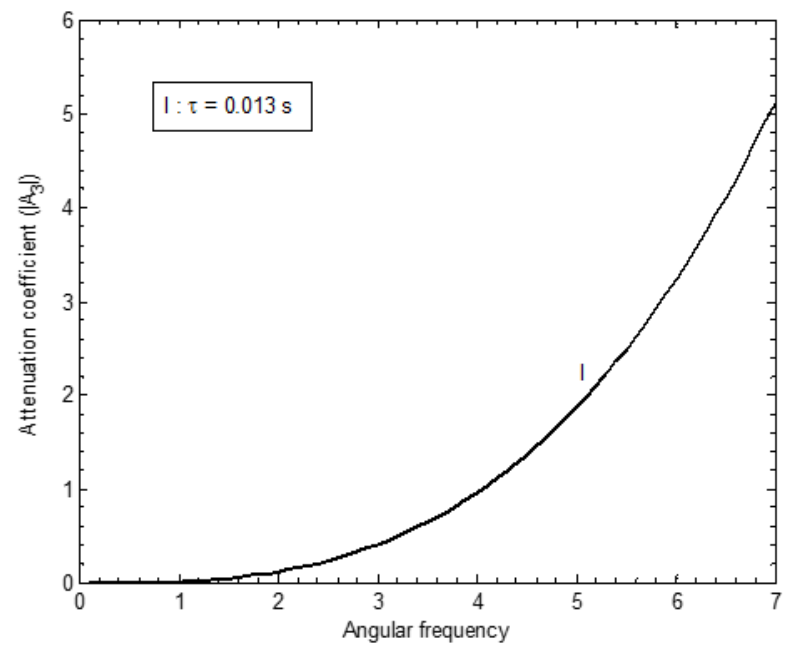

Figure 6 | Attenuation coefficient of shear wave $\left(\left|A_{3}\right|\right)$.

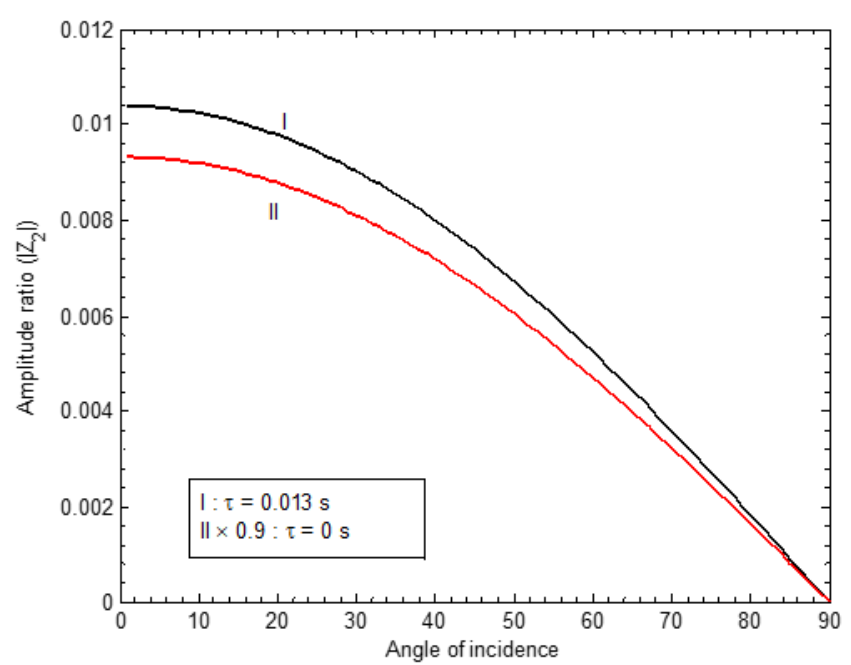

Figure 8 | Amplitude ratio of slow longitudinal wave $\left(\left|Z_{2}\right|\right)$.

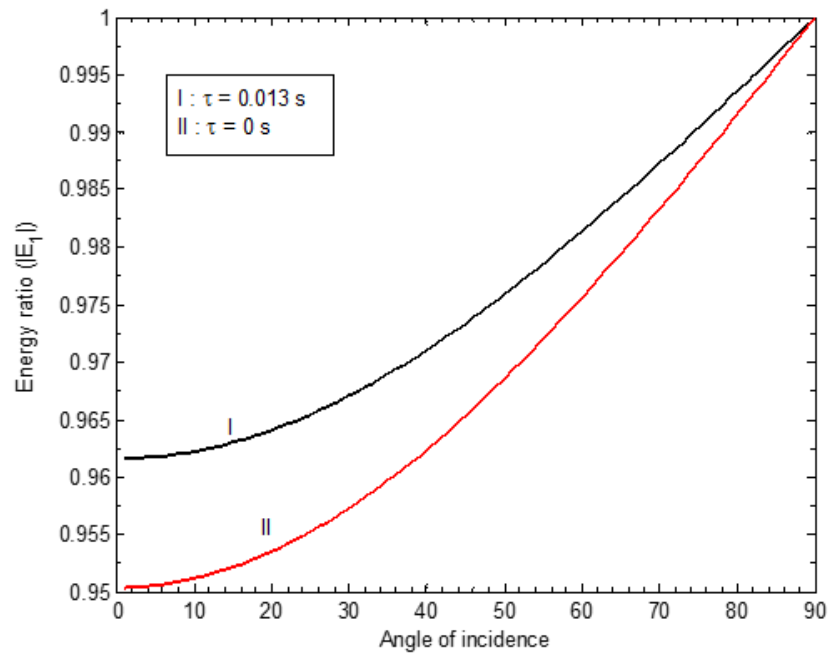

Figure 10 | Energy ratio of fast longitudinal wave $\left(\left|E_{1}\right|\right)$.

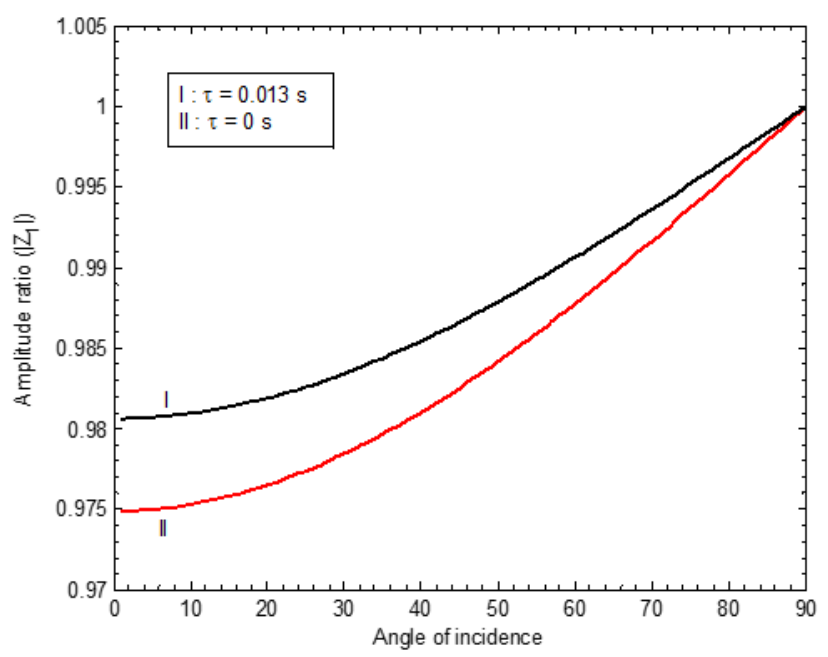

Figure 7 | Amplitude ratio of fast longitudinal wave $\left(\left|Z_{1}\right|\right)$.

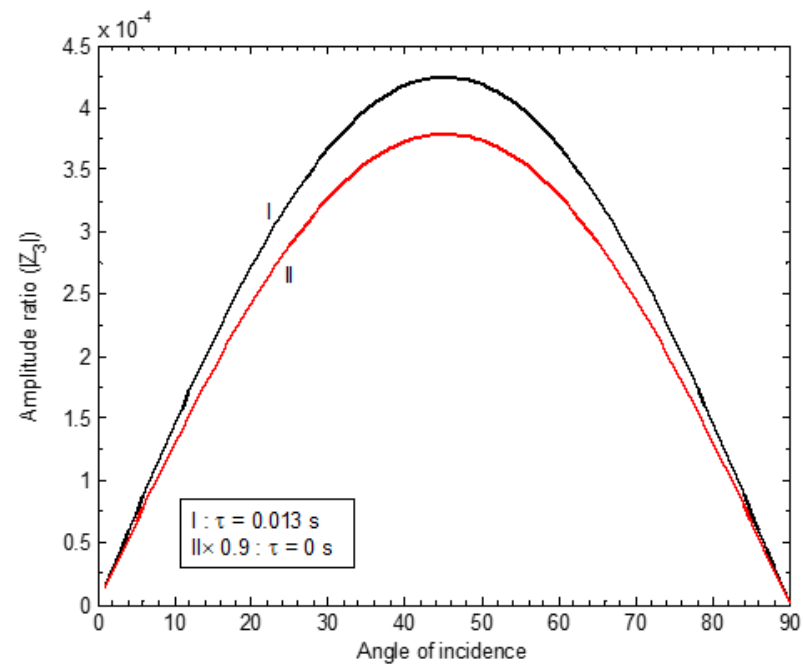

Figure 9 | Amplitude ratio of shear wave $\left(\left|Z_{3}\right|\right)$.

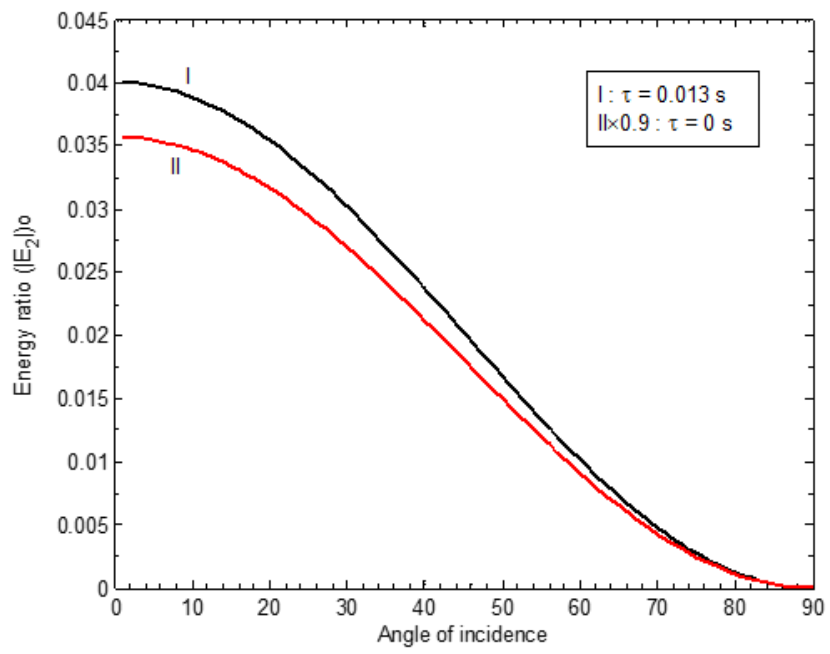

Figure 11 | Energy ratio of slow longitudinal wave (|E $\left.E_{2} \mid\right)$. 


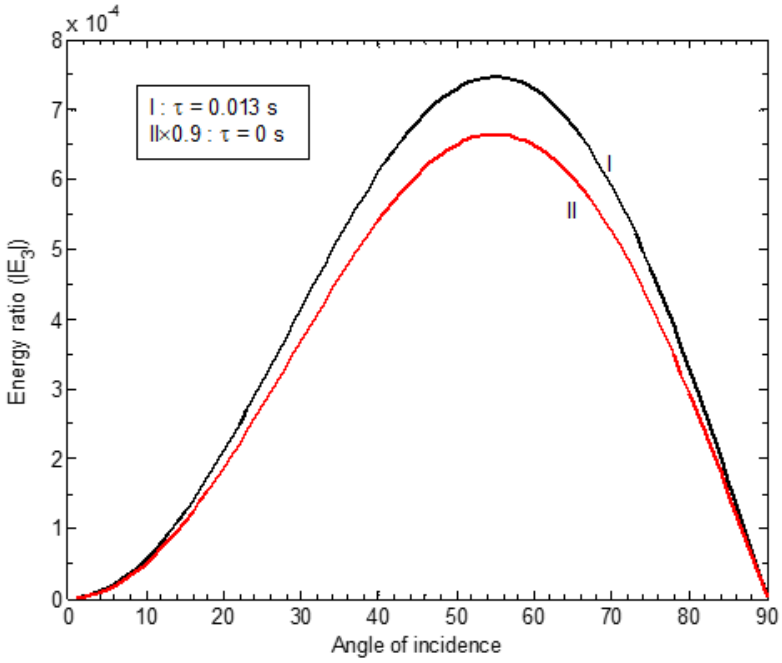

Figure 12 | Energy ratio of shear wave $\left(\left|E_{3}\right|\right)$.

- The phase speeds in the absence of mechanical relaxation time has higher value than the normal mechanical relaxation time, while the shear wave has higher value than the absence of mechanical relaxation time.

- The similar effect of mechanical relaxation time is found for the corresponding attenuation coefficient while the shear wave is nonattenuate in the absence of mechanical relaxation time.

- The amplitude and energy ratios have shown larger values in the presence of mechanical relaxation time then the absence of it.

- The sum of energy ratios is closed to unity both in the cases $(\tau=0$ and $\tau=0.013 \mathrm{~s}$ ).

The present investigation and observation may be useful in the study of wave propagation in the related materials medium containing voids. It may also be useful in the fields of geology and seismology.

\section{Conflict of interest}

None declared.

\section{References}

1. Cowin, S. C., Nunziato, J. W. (1983). Linear theory of elastic materials with voids. Journal of Elasticity, 13, 125-147. https://doi.org/10.1007/BF00041230

2. Puri, P., Cowin, S. C. (1985). Plane waves in linear elastic materials with voids. Journal of Elasticity, 16, 167-183. https://doi.org/10.1007/BF00041991

3. Chanrasekhariah, D. S. (1987). A uniqueness theorem in the theory of elastic materials with voids. Journal of Elasticity, 18, 173-179. https:// doi.org/10.1007/BF00127556.

4. Iovane, G., Nasedkin, A. V., (2007). Some theorems about spectrum and finite element approach for eigenvalue problems for elastic bodied with voids. Computers and Mathematics with Application, 53, 789 -802. https://doi.org10.1016/j.camwa.2007.01.003

5. Scalia, A. (1994). Shock waves in viscoelastic materials with voids. Wave Motion, 19, 125-133. https://doi.org/10.1016/0165-2125(94)90061-2

6. Chirila, A. (2017). Generalized micropolar thermoelasticity with fractional order strain. Bulletin of the Transilvania University of Brasov, 10, 83-90.

7. Scalia, A., Sumbatyan, M. A. (2000). Contact problem for porous elastic half-plane. Journal of Elasticity, 60, 91-102. https://doi.org/10.1023/ A:1010880823544

8. Ciarletta, M., Sumbatyan, M. A. (2003). Reflection of plane waves by the free boundary of porous elastic half-space. Journal of Sound and Vibration, 259, 253-264. http://doi.org/10.1006/jsvi.2002.5149

9. Tomar, S. K., Singh, J. (2005). Transmission of longitudinal waves through a plane interface between two dissimilar porous elastic solid halfspace. Applied Mathematics and Computation, 169, 671-688. https://doi.org/10.1016/j.amc.2004.09.060

10. Singh, J., Tomar, S. K. (2005). Reflection and transmission of transverse wave at a plane interface between two different porous solid half spaces. Applied Mathematics and Computation, 176, 364-367. https://doi.org/10.1016/j.acm.2005.09.027

11. Chandrasekhariah, D. S. (1987). Effect of surface stresses and voids on Rayleigh waves in an elastic solid. International Journal of Engineering Science, 25, 205-211. https://doi.org/10.1016/0020-7225(87) 90006-1

12. Chandrasekhariah, D. S. (1987). Rayleigh-Lamb waves in an elastic plate with voids. Journal of Applied Mechanics, 54, 509-512. https:// doi.org/10.1115/1.3173061

13. Abo-Dahab, S. M., Singh, B. (2013). Rotational and voids effect on the reflection of $P$ waves from stress-free surface of an elastic half-space under magnetic field and initial stress without energy dissipation. Applied Mathematical Modelling, 37, 8999-9011. https://doi.org/10.1016/ j.apm.2013.04.033

14. Dey, S., Gupta, S., Gupta, A. K. (1993). Torsional surface wave in an elastic half-space with void pores. International Journal for Numerical Analytical 
Method in Geomechanics, 17, 197-204. https:// doi.org/10.1002/nag.1610170305

15. Chirita, S., Ghiba, I. D. (2010). Inhomogeneous plane waves in elastic materials with voids. Wave Motion, 47, 333-342. https://doi.org/10.1016/ j.wavemoti.2010.01.003

16. Achenbach, J. D. (1976). Wave propagation in elastic solid, North-Holland Publishing Company, New York, pp. 1-425.

17. Straughan, B. (2009). Nonlinear acceleration waves in porous media. Mathematics and Computers in Simulation, 80, 763-769. https://doi.org/10.1016/ j.matcom.2009.08.013

18. Lianngenga, R. (2015). Voids effect on the propagation of elastic waves in a micropolar medium. Science Vision, 15, 185-190.
19. Lianngenga, R. (2017). Effect of inertial coefficients in the propagation of plane waves in micropolar porous materials. International Journal of Computational Materials Science and Engineering, 6, 1750007(1-16). https://doi.org/10.1142/ S2047684117500075

20. Lianngenga, R., Lalawmpuia, Lalvohbika, J. (2015). Phase velocities of elastic waves in swelling porous materials. Science Vision, 15, 68-75.

21. Lianngenga, R., Duhawma, L. P., Thangmawia, L. (2018). Symmetric and antisymmetric vibration in elastic plate with voids immersed in a liquid. International Journal of Computational Materials Science and Engineering, 7, 1850017(1-13). https:// doi.org/10.1142/S2047684118500173

22. Ciarletta, M., Iesan, D. (1993). Non-Classical Elastic Solids. Longman Scientific and Technical, Harlow, Essex, pp. 1-360. 